\title{
Hybrid stars with large strange quark cores constrained by GW170817
}

\author{
Márcio Ferreira,$^{*}$ Renan Câmara Pereira $\odot,{ }^{\dagger}$ and Constança Providência $\odot^{*}$ \\ CFisUC, Department of Physics, University of Coimbra, P-3004-516 Coimbra, Portugal
}

(Received 30 March 2021; accepted 25 May 2021; published 22 June 2021)

\begin{abstract}
We analyze the impact of several multiquark interaction channels on the properties of hybrid stars. Four and eight vector interactions were included in the $S U(3)$ Nambu-Jona-Lasinio model to describe the quark matter, and all vectorlike interactions were investigated. The hybrid stars were built starting from an hadronic equation of state that satisfy presently accepted nuclear matter properties and considering the quark model constrained by the vacuum properties of several light mesons. The interplay between the eight-quark vector interaction and the four-quark isovector-vector interaction determine the size of the quark core and its strangeness content. The inclusion of a isovector-vector interaction is essential to obtain large $s$-quark contents and a smaller central speed of sound, although this interaction pushes the onset of quarks to larger densities. It is observed that low mass stars, with a mass below $1.4 M_{\odot}$, may contain a quark core but with a small strangeness content. It is shown that the presently existing mass and tidal deformability constraints from neutron stars observations allow for the existence of hybrid stars with a large strangeness content and large quark cores for binary mass ratios $q=M_{2} / M_{1} \gtrsim 0.85$.

DOI: 10.1103/PhysRevD.103.123020
\end{abstract}

\section{INTRODUCTION}

Multimessenger astrophysics provides deeper insights into neutron stars (NS) physics by combining astrophysical observations of electromagnetic radiation and gravitational waves (GW). The observation of the pulsars PSR J1614$2230\left(M=1.908 \pm 0.016 M_{\odot}\right)[1,2]$, PSR J0348 +0432 $\left(M=2.01 \pm 0.04 M_{\odot}\right)$ [3], and MSP J0740 + 6620 [4], $\left(M=2.14_{-0.09}^{+0.10} M_{\odot}\right)$ established considerable constraints on the nuclear matter equation of state $(\mathrm{EoS})$. The analysis by the LIGO/Virgo Collaboration of the GW170817 NS merger provided important information about NS physics $[5,6]$, e.g., an upper limit of the tidal deformability of a NS star, constraining the high density EoS. The observations of the gamma-ray burst (GRB) GRB170817A [7] and electromagnetic transient AT2017gfo [8], which followed up the GW170817 event, give further hints on the NS tidal deformability [9-13]. A recent observation from the LIGO/Virgo Collaboration is the compact binary coalescence GW190814 [14]. Even though the primary binary component is conclusively a black hole of mass 22.2-24.3 $M_{\odot}$, the nature of the secondary component with a mass $2.50-2.67 M_{\odot}$ remains yet inconclusive [14]. Recent estimates for both the mass and radius of the millisecond-pulsar PSR J0030 + 0451 were put forward by the Neutron Star Interior

\footnotetext{
*marcio.ferreira@uc.pt renan.pereira@student.uc.pt cp@fis.uc.pt
}

Composition Explorer (NICER) mission [15]. However, the uncertainties obtained are still large and therefore, do not constrain much the EoS.

The NS tidal deformability estimated from GW observations favors a not too stiff EoS for NS matter [16]. However, this behavior conflicts with the observations of massive pulsars, which require a stiff EoS and predict large radii and thus large tidal deformabilities. The contrasting indications of low radii predictions from tidal deformabilities estimations and large radii from massive pulsar observations pose considerable constraints on the high density region of the nuclear matter EoS. These indications might be a signal of the existence of nonnucleonic degrees of freedom at high densities in NS matter, such as quark matter. The existence of a firstorder phase transition from hadronic to quark matter may still explain the observational data [17]. Detecting observational signatures for the presence of exotic matter inside NS is a major difficulty; e.g., it is hard to distinguish between a purely hadronic NS and one with a quark core solely from NS observables, such as the star mass, radius and tidal deformability. However, the presence of a first-order phase transition to quark matter can imprint signatures in NS binary mergers observations, favoring the hypothesis of quark matter in the NS core [17-19].

QCD effective models that incorporate important properties and symmetries of the strong interaction are widely used for describing quark degrees of freedom in NS matter. The Nambu-Jona-Lasinio (NJL) model is one of such 
effective models that considers chiral symmetry preserving interactions $[20,21]$ and that has been used to study the possible existence of quark matter inside NS [22-31].

The description of NS matter with non-nucleonic degrees of freedom is achieved by constructing a hybrid EoS. A possible method to obtain the EoS is to consider a twomodel approach: one that describes the hadronic (confined) phase and a second model describing the quark (deconfined) phase. While different procedures can be used for the matching of the two EoS [32], we consider a Maxwell construction in order to describe a first-order phase transition from hadron to quark mater. The reliability of such approach depends on the still unknown surface tension between both phases; however, it is justified and has been widely considered [26,33-37], if the surface tension, $\sigma$, is of the order of $\sigma \gtrsim 40 \mathrm{MeV} \mathrm{fm}^{-2}$ [38]. The NJL model has been explored in describing the quark phase of a hybrid EoS in several works, e.g., [27,36,39]. The presence of the vector-isoscalar interactions in the NJL model was shown to be very important in stiffening the EoS to sustain $2 M_{\odot}$. The eight-quark interactions were first introduced to stabilize the NJL vacuum of the four-quark NJL and six-quark 't Hooft interactions [40,41]. The study of the effect of scalar and vector eight-quark terms in stellar matter was done in [33], where it was shown that these terms allow for the description of two solar mass hybrid stars, with the eightquark scalar channel giving rise to a reduction of the onset of quark matter, and the eight-quark vector channel stiffening the equation of state at high densities. Within this same model it was possible to describe twin stars [34] considering a very stiff hadronic EoS. In $[42,43]$ further studies were undertaken with the same model, in particular, a Bayesian analysis which studied the possibility of discriminating a hybrid EoS with a strong first order phase transition over other possible EoS [42]. It was also studied the possible existence of a critical end point in the QCD phase diagram, taking into account the $2 M_{\odot}$ NS constraint [43].

Local and nonlocal versions of NJL models including the four-quark vector interaction typically would not predict the existence of hybrid stars (or just small quark branches) $[22,25,44]$. Other approaches for describing quark matter in NS were also explored. In $[45,46]$, the high-density EoS region was analyzed by a constant speed of sound parametrization, showing that a large speed of sound in the quark phase, $v_{s}^{2} \gtrsim 0.5$ for soft hadronic EoS and $v_{s}^{2} \gtrsim 0.4$ for stiff hadronic EoS would be required for $M_{\max }>2 M_{\odot}$. Using the same formalism, [47] showed that strong repulsive interactions in quark matter are required to support the NS masses $M \gtrsim 2.0 M_{\odot}$. In [48], imposing constraints from observational and theoretical $a b$ initio calculations, the authors proposed that $1.4 M_{\odot} \mathrm{NS}$ are compatible with hadronic stars, by using a speed of sound EoS parametrization. Furthermore, the authors inferred that massive stars $\approx 2 M_{\odot}$ and a speed of sound not far from the conformal limit will have large quark cores.

In our previous works [49,50], we have analyzed hybrid stars using the NJL model with different interaction terms at the Lagrangian level which describes the quark phase. The usual three-flavor NJL model [20,51] with additional vector and pseudovector interactions and the vector-isovector and pseudovector-isovector interactions were explored in [49]. It was shown that considerable quark core sizes would require moderate values for the quark vector-isoscalar term and a weak vector isovector term. Additional higher-order repulsive interactions, four-quark and eight-quark vector-isoscalar interactions, were included in [50]. The eight-quark vector-isoscalar channel was shown to allow for the appearance of a quark core at moderately low NS masses, $\sim 1 M_{\odot}$, while providing the required repulsion to keep the star stability up to $\sim 2.1 M_{\odot}$. It was also shown that both the heaviest NS mass and radius are sensitive to the strength of an eight-quark vectorisoscalar channel [50].

The present work is an extension of our previous works: we explore the effect of all the four-quark and eight-quark vector interaction channels within the threeflavor NJL model in describing hybrid EoS. We will investigate the impact of the interaction channels in the existence and stability of hybrid star sequences and on the quark core properties, such as mass and radius. The possibility of having quark cores in light NS and fulfilling all observational constraints will be studied. In particular, we will look at the strangeness content and analyse whether NS with large quark cores may have a high $s$-quark fraction. Immediately after the measurement of the mass of the pulsar PSR J1614-2230, it was suggested that exotic degrees of freedom such as strangeness might be ruled out with the detection of a two solar mass NS [1,52]. However, since then, several studies have shown that in fact it is possible that hyperons nucleate inside a star with at least two solar masses [53-58]; see [59] for a review. Also inside hybrid stars, the existence of strange quarks was not ruled out by the two solar mass constraint; see for instance $[27,36,60]$. Recently, within a Bayesian analysis, which incorporates both information from the GW170817 binary neutron star merger $[5,6]$, the MP J0740 + 6620 [4] and the pulsar PSR J0030+ 0451 as detected by NICER [15], it was shown that the maximum mass of an hybrid star could be above $2 M_{\odot}[61]$.

This paper is organized as follows: in Sec. II the quark model is presented. The results are discussed in Sec. III and our conclusions are drawn in Sec. IV.

\section{MODEL AND FORMALISM}

For the quark matter, we consider the following multiquark interaction Lagrangian density: 


$$
\begin{aligned}
\mathcal{L}= & \bar{\psi}\left(i \not \partial-\hat{m}+\hat{\mu} \gamma^{0}\right) \psi+G_{S} \sum_{a=0}^{8}\left[\left(\bar{\psi} \lambda^{a} \psi\right)^{2}+\left(\bar{\psi} i \gamma^{5} \lambda^{a} \psi\right)^{2}\right]-G_{D}\left[\operatorname{det}\left(\bar{\psi}\left(1+\gamma_{5}\right) \psi\right)+\operatorname{det}\left(\bar{\psi}\left(1-\gamma_{5}\right) \psi\right)\right] \\
& -G_{\omega}\left[\left(\bar{\psi} \gamma^{\mu} \lambda^{0} \psi\right)^{2}+\left(\bar{\psi} \gamma^{\mu} \gamma_{5} \lambda^{0} \psi\right)^{2}\right]-G_{\rho} \sum_{a=1}^{8}\left[\left(\bar{\psi} \gamma^{\mu} \lambda^{a} \psi\right)^{2}+\left(\bar{\psi} \gamma^{\mu} \gamma_{5} \lambda^{a} \psi\right)^{2}\right]-G_{\omega \omega}\left[\left(\bar{\psi} \gamma^{\mu} \lambda^{0} \psi\right)^{2}+\left(\bar{\psi} \gamma^{\mu} \gamma_{5} \lambda^{0} \psi\right)^{2}\right]^{2} \\
& -G_{\rho \rho} \sum_{a=1}^{8} \sum_{b=1}^{8}\left[\left(\bar{\psi} \gamma^{\mu} \lambda^{a} \psi\right)^{2}+\left(\bar{\psi} \gamma^{\mu} \gamma_{5} \lambda^{a} \psi\right)^{2}\right]\left[\left(\bar{\psi} \gamma^{\mu} \lambda^{b} \psi\right)^{2}+\left(\bar{\psi} \gamma^{\mu} \gamma_{5} \lambda^{b} \psi\right)^{2}\right] \\
& -G_{\omega \rho}\left[\left(\bar{\psi} \gamma^{\mu} \lambda^{0} \psi\right)^{2}+\left(\bar{\psi} \gamma^{\mu} \gamma_{5} \lambda^{0} \psi\right)^{2}\right] \sum_{a=1}^{8}\left[\left(\bar{\psi} \gamma^{\mu} \lambda^{a} \psi\right)^{2}+\left(\bar{\psi} \gamma^{\mu} \gamma_{5} \lambda^{a} \psi\right)^{2}\right] .
\end{aligned}
$$

This is a SU(3) NJL-type model that includes four and six scalar-pseudoscalar interactions, and four and eight vector interactions. The quark current masses and chemical potentials are given by $\hat{m}=\operatorname{diag}\left(m_{u}, m_{d}, m_{s}\right)$ and $\hat{\mu}=\operatorname{diag}\left(\mu_{u}, \mu_{d}, \mu_{s}\right)$, respectively. The model is regularized by a three-momentum cutoff $\Lambda$. We note that the Lagrangian density terms designated with a subscript $\rho$ involve the three flavors, $u, d$ and $s$ and therefore, are hypercharge operators.

The four scalar and pseudoscalar quark interaction are present in the original formulation of the NJL model and are essential to incorporate spontaneous chiral symmetry breaking in the model. The 't Hooft determinant for three quark flavors corresponds to a six quark interaction which incorporates the explicit $U_{A}(1)$ symmetry breaking in the model. Incorporating vector interaction in the model has been found to be necessary to model the medium to high density behavior of the EoS and predict $2 M_{\odot}$ NSs. The inclusion of all possible chiral-symmetric set of eight quark vector interactions was performed in [62], in order to study the masses of the lowest spin- 0 and spin- 1 meson states.

In the mean-field approximation, the model thermodynamical potential is given by

$$
\begin{aligned}
\Omega-\Omega_{0}= & 2 G_{S}\left(\sigma_{u}^{2}+\sigma_{d}^{2}+\sigma_{s}^{2}\right)-4 G_{D} \sigma_{u} \sigma_{d} \sigma_{s}-\frac{2}{3} G_{\omega}\left(\rho_{u}+\rho_{d}+\rho_{s}\right)^{2}-G_{\rho}\left(\left(\rho_{u}-\rho_{d}\right)^{2}+\frac{1}{3}\left(\rho_{u}+\rho_{d}-2 \rho_{s}\right)^{2}\right) \\
& -\frac{4}{3} G_{\omega \omega}\left(\rho_{u}+\rho_{d}+\rho_{s}\right)^{4}-3 G_{\rho \rho}\left(\left(\rho_{u}-\rho_{d}\right)^{2}+\frac{1}{3}\left(\rho_{u}+\rho_{d}-2 \rho_{s}\right)^{2}\right)^{2} \\
& -2 G_{\omega \rho}\left(\rho_{u}+\rho_{d}+\rho_{s}\right)^{2}\left(\left(\rho_{u}-\rho_{d}\right)^{2}+\frac{1}{3}\left(\rho_{u}+\rho_{d}-2 \rho_{s}\right)^{2}\right) \\
& -2 N_{c} \sum_{i=u, d, s} \int \frac{d^{3} p}{(2 \pi)^{3}}\left[E_{i}+T \ln \left(1+e^{-\left(E_{i}+\tilde{\mu}_{i}\right) / T}\right)+T \ln \left(1+e^{-\left(E_{i}-\tilde{\mu}_{i}\right) / T}\right)\right],
\end{aligned}
$$

where $E_{i}=\sqrt{p^{2}+M_{i}^{2}}, \sigma_{i}$ and $\rho_{i}$ are the $i$-flavor quark condensate and quark density. The constant $\Omega_{0}$ is fixed to give a vanishing vacuum potential. The values for the quark condensates, $\sigma_{i}$, and densities, $\rho_{i}$, are obtained by requiring the potential to be stationary with respect to the effective masses and effective chemical potentials, $\partial \Omega / \partial M_{i}=0$ and $\partial \Omega / \partial \tilde{\mu}_{i}=0$ i.e., imposing thermodynamic consistency [21]. In the mean-field approximation, the product between quark bilinear operators in Eq. (1) is linearized, providing an effective Lagrangian which is quadratic in the fermion fields. Using such approximation, it is possible to obtain the thermodynamical potential written in Eq. (2). For more details on the linear expansion of the product between $N$ operators, see [63]. For $i \neq j \neq k \in\{u, d, s\}$, the effective mass, $M_{i}$, and effective chemical potentials, $\tilde{\mu}_{i}$, are given by

$$
M_{i}=m_{i}-4 G_{S} \sigma_{i}+2 G_{D} \sigma_{j} \sigma_{k}
$$

$$
\begin{aligned}
\tilde{\mu}_{i}= & \mu_{i}-\frac{4}{3} G_{\omega}\left(\rho_{i}+\rho_{j}+\rho_{k}\right)-\frac{4}{3} G_{\rho}\left(2 \rho_{i}-\rho_{j}-\rho_{k}\right) \\
& -\frac{16}{9} G_{\omega \omega}\left(\rho_{i}+\rho_{j}+\rho_{k}\right)^{3} \cdot-\frac{32}{9} G_{\rho \rho}\left(2 \rho_{i}-\rho_{j}-\rho_{k}\right)^{3} \\
& -\frac{32}{3} G_{\rho \rho}\left(\rho_{i}-\rho_{j}\right)\left(\rho_{k}-\rho_{i}\right)\left(2 \rho_{i}-\rho_{j}-\rho_{k}\right) \\
& -\frac{8}{9} G_{\omega \rho}\left(\rho_{i}+\rho_{j}+\rho_{k}\right) \\
& \times\left(4 \rho_{i}^{2}+\rho_{j}^{2}+\rho_{k}^{2}-\rho_{i} \rho_{j}-\rho_{i} \rho_{k}-4 \rho_{j} \rho_{k}\right)
\end{aligned}
$$

By taking the zero temperature limit in Eq. (2), one can determine the pressure and energy density for cold quark matter $P=-\Omega$ and $\epsilon=-P+\sum_{i} \mu_{i} \rho_{i}$, respectively. In the following analysis, we will represent the EoS quantities as a function of baryonic density, $n$, which is given by $n=\left(\rho_{u}+\rho_{d}+\rho_{s}\right) / 3$. 
TABLE I. Parameters of the NJL model used in the present work: $\Lambda$ is the model cutoff, $m_{u, d}$ and $m_{s}$ are the quark current masses, $G_{S}$ and $G_{D}$ are coupling constants. $M_{u, d}$ and $M_{s}$ are the resulting constituent quark masses in the vacuum. This parameter set yields, in the vacuum, a light quark condensate of $\left\langle\bar{q}_{l} q_{l}\right\rangle^{1 / 3}=-243.9 \mathrm{MeV}$ and strange quark condensate of $\left\langle\bar{q}_{s} q_{s}\right\rangle^{1 / 3}=-262.9 \mathrm{MeV}$.

\begin{tabular}{lcccccr}
\hline \hline$\Lambda[\mathrm{MeV}]$ & $m_{u, d}[\mathrm{MeV}]$ & $m_{s}[\mathrm{MeV}]$ & $G_{S} \Lambda^{2}$ & $G_{D} \Lambda^{5}$ & $M_{u, d}[\mathrm{MeV}]$ & $M_{s}[\mathrm{MeV}]$ \\
\hline 623.58 & 5.70 & 136.60 & 1.67 & 13.67 & 332.2 & 510.7 \\
\hline \hline
\end{tabular}

TABLE II. The masses and decay constants of several mesons within the model and the respective experimental values.

\begin{tabular}{lcc}
\hline \hline & NJL SU(3) & Experimental [64] \\
\hline$m_{\pi^{ \pm}}[\mathrm{MeV}]$ & 139.6 & 139.6 \\
$f_{\pi^{ \pm}}[\mathrm{MeV}]$ & 92.0 & 92.2 \\
$m_{K^{ \pm}}[\mathrm{MeV}]$ & 493.7 & 493.7 \\
$f_{K^{ \pm}}[\mathrm{MeV}]$ & 96.4 & 110.4 \\
$m_{\eta}[\mathrm{MeV}]$ & 515.6 & 547.9 \\
$m_{\eta^{\prime}}[\mathrm{MeV}]$ & 957.8 & 957.8 \\
\hline \hline
\end{tabular}

The model interaction couplings include the usual NJL parameters $\left\{G_{S}, G_{D}, \Lambda\right\}$ and the following additional extra terms $\left\{G_{\omega}, G_{\omega \omega}, G_{\rho}, G_{\rho \rho}, G_{\omega \rho}\right\}$. Our goal is to analyze the overall effect of the additional terms on the NS properties of hybrid EoS. For that, we fix $G_{S}, G_{D}$, and $\Lambda$ to reproduce the meson masses of the $\pi^{ \pm}, K^{ \pm}, \eta$ and $\eta^{\prime}$ and the leptonic decay constants of the $\pi^{ \pm}$and $K^{ \pm}, f_{\pi^{ \pm}}$and $f_{K^{ \pm}}$, while leaving the extra coupling as free parameters. We show the parameter set used in Table I, and the model predictions, within the present parametrization, for some meson masses and leptonic decay constants in Table II.

As in our previous works [36], each model parametrization is identified by dimensionless ratios instead of the actual couplings values. The quark models are characterized by the following five coupling ratios: $\chi_{\omega}=G_{\omega} / G_{S}$, $\chi_{\omega \omega}=G_{\omega \omega} / G_{S}^{4}, \quad \chi_{\rho}=G_{\rho} / G_{S}, \quad \chi_{\rho \rho}=G_{\rho \rho} / G_{S}^{4}, \quad$ and $\chi_{\omega \rho}=G_{\omega \rho} / G_{S}^{4}$. In the following, we identify each model by the set of values $\left\{\chi_{\omega}, \chi_{\omega \omega}, \chi_{\rho}, \chi_{\rho \rho}, \chi_{\omega \rho}\right\}$. The model pressure, $P$, and energy density, $\epsilon$, are defined up to an extra constant term $B$, i.e., $P \rightarrow P+B$ and $\epsilon \rightarrow \epsilon-B$. The effect of the bag parameter $B$ was already widely studied $[23,26-$ $31,36,49,65-67]$. It was found that the baryonic density at which the onset of quark matter occurs decreases with increasing $B$. We have verified that this behavior remains the same in the presence of any of the considered interactions and regardless of their coupling values. Therefore, for the sake of simplicity, we fix, hereafter, the bag parameter to $B=15 \mathrm{MeV} / \mathrm{fm}^{3}$.

Each hybrid EoS consists of a hadronic phase connected to a quark phase through a first-order phase transition. This

\footnotetext{
${ }^{1}$ There was a misprint in the $B$ value reported in our previous work [50], being $B=15 \mathrm{MeV} / \mathrm{fm}^{3}$ the value used instead of the reported value, $B=10 \mathrm{MeV} / \mathrm{fm}^{3}$.
}

two-model approach has been widely used [26,33-37]. The first-order phase transition from hadronic to quark matter is achieved imposing the Maxwell construction, in which both phases are in chemical, thermal and mechanical equilibrium: $\mu_{B}^{H}=\mu_{B}^{Q}, P^{H}=P^{Q}$, and $T^{H}=T^{Q}$, where $\mu_{B}$ is the baryon chemical potential, $P$ the pressure and the labels $H$ and $Q$ represent the hadronic and quark phases, respectively. The DDME2 model is used for the hadronic part [68]. This is a relativistic mean-field model with density dependent couplings that describes two solar mass stars and satisfies a well established set of nuclear matter and finite nuclei constraints [58,69], including the constraints set by the $a b$ initio calculations for neutron matter using a chiral effective field theoretical approach [70]. It is important to realize that the hadronic EoS also has an impact in the hybrid star EoS: having a softer EoS, for example, would shift the deconfinement transition to larger densities, giving rise to smaller quark branches, and to the possibility of not fulfilling the $2 M_{\odot}$ star constraint. The opposite would be valid for a harder hadronic EoS. In [49] we have shown that, in order to get $2 M_{\odot}$ hybrid stars, the hadronic EoS has to be in average stiffer than necessary for a $2 M_{\odot}$ hadronic star. Having this in mind, we chose for the hadronic phase an EoS that would allow $2 M_{\odot}$ hybrid stars and still satisfy well established nuclear properties.

\section{RESULTS}

The aim of the present work is to analyze the effect of quark matter on the hybrid stars properties. The quark model we are considering has five free parameters: $\chi_{\omega}=G_{\omega} / G_{S}, \chi_{\omega \omega}=G_{\omega \omega} / G_{S}^{4}, \chi_{\rho}=G_{\rho} / G_{S}, \chi_{\rho \rho}=G_{\rho \rho} / G_{S}^{4}$, $\chi_{\omega \rho}=G_{\omega \rho} / G_{S}^{4}$. In our previous works [49,50], we have already analyzed some interactions of the present quark model [Eq. (1)], which we briefly summarize. The couplings $\left\{\chi_{\omega}, \chi_{\rho}\right\}$ were analyzed in [49], where a set of hadronic EoS was used. In there, the $\chi_{\omega}$ coupling was seen to stiffen the quark EoS, with the onset of quarks occurring at larger densities, giving rise to more massive hybrid stars with smaller quark cores. Similar results were also discussed in $[31,36,50]$. On the other hand, $\chi_{\rho}$ softens the quark EoS at higher densities, as the onset of the strange quarks is pushed to lower densities but stiffens the quark EoS at lower densities while the $s$-quark does not set in. As a result, the onset of quark matter occurs at higher densities for a nonzero $\chi_{\rho}$, and sizeable quark cores are only obtained 


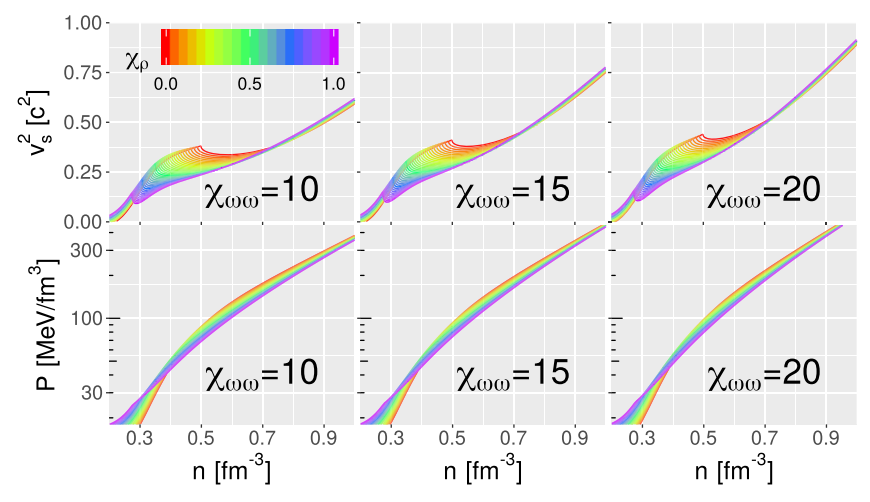

FIG. 1. Quark matter squared speed of sound (top) and pressure (bottom) as function of baryon density for different values of $\chi_{\rho}$ with $\chi_{\omega \omega}=10$ (left), $\chi_{\omega \omega}=15$ (center), and $\chi_{\omega \omega}=20$ (right).

if $\chi_{\rho}<0.4[36,49]$. The effect of the $\left\{\chi_{\omega}, \chi_{\omega \omega}\right\}$ interactions was the purpose of our work [50]. Besides the effect of $\chi_{\omega}$ we just referred, it was also shown that $\chi_{\omega \omega}$ induces a nonlinear density dependence of the speed of sound, which is crucial to generate large quark cores.

Despite controlling the onset density of strange quarks, the impact of both $\chi_{\rho \rho}$ and $\chi_{\omega \rho}$ couplings on the EoS quark matter in $\beta$-equilibrium is weak when compared with the other couplings. Almost all NS properties show small changes with both couplings and, for the sake of simplicity, we fix them to $\chi_{\rho \rho}=\chi_{\omega \rho}=0$ hereafter. While a detailed study of both couplings is left for future work, we give later a brief overview of their impact. The effect of $\chi_{\omega}$ was confirmed; i.e., the onset of quark matter happens at larger densities with increasing coupling, giving rise to smaller quark cores. In a first approach, we consider $\chi_{\omega}=0$ and analyze the impact of $\left\{\chi_{\omega \omega}, \chi_{\rho}\right\}$ on the properties of hybrid stars. In a second step, we consider the interplay among the three couplings, $\left\{\chi_{\omega \omega}, \chi_{\rho}, \chi_{\omega}\right\}$. In fact, as expected, a nonzero $\chi_{\omega}$ changes the results as just referred, e.g., a finite $\chi_{\omega}$ gives more massive stars with smaller quark cores.

\section{A. Quark matter EOS}

We first discuss the joint effect of the couplings $\left\{\chi_{\omega \omega}, \chi_{\rho}\right\}$ on the properties of the EoS and set $\chi_{\omega}=0$. Figure 1 shows the squared speed of sound (top panels) and the pressure (bottom panels) of the quark EoS as a function of baryonic density for different values of $\chi_{\rho}$ (color scale) and $\chi_{\omega \omega}$ (different panels). As $\chi_{\omega \omega}$ increases, the pressure, $P(n)$ increases at high densities: the EoS clearly shifts to larger values of $P$. The increase of the $\chi_{\rho}$ coupling shifts the onset of strange quarks to lower densities with direct consequences on the stiffness of the EoS: a) at low densities, below the $s$-quark onset, the larger the value of $\chi_{\rho}$ the stiffer the EoS; b) the EoS smooths as soon as the $s$-quark sets in, and this happens first to the large values of $\chi_{\rho}$ (purple color), and above this onset density the EoS with larger values of $\chi_{\rho}$ have the smaller pressures. The $v_{s}^{2}(n)$

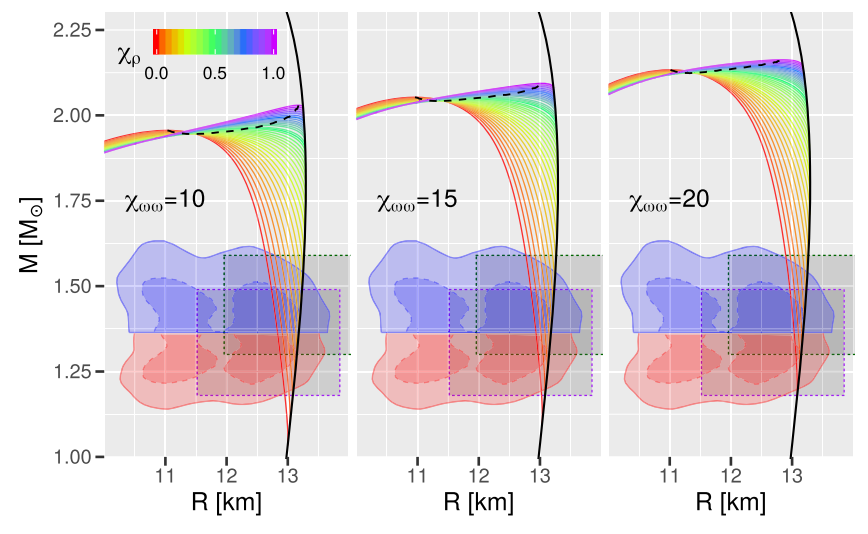

FIG. 2. $M(R)$ diagrams for $\chi_{\omega \omega}=10$ (left), $\chi_{\omega \omega}=15$ (center), and $\chi_{\omega \omega}=20$ (right). The color scale indicates the $\chi_{\rho}$ value, and the solid black line represents the purely hadronic sequence. The black dashed line indicates the maximum mass reached by each EoS. The top blue and bottom red regions indicate, respectively, the $90 \%$ (solid) and 50\% (dashed) credible intervals of the LIGO/ Virgo analysis for each binary component from the GW170817 event [74] (using a set of parametrized EoS that assumes $M_{\max } \geq 1.97 M_{\odot}$ ). The rectangular regions enclosed by dotted lines indicate the constraints from the millisecond pulsar PSR J0030 + 0451 NICER x-ray data $[15,75]$.

reflects this behavior: the sudden decrease between 0.3 and $0.5 \mathrm{fm}^{-3}$ signals the appearance of strange quarks which moves towards lower densities with increasing $\chi_{\rho}$. A smoother crossover to strange matter is obtained with smaller values of $\chi_{\rho}$ because the fraction of $s$-quarks increases more slowly; see [36].

For the highest densities, $v_{s}^{2}(n)$ is again larger for the larger $\chi_{\rho}$ couplings, revealing the stiffening effect of $\chi_{\rho}$ once the fraction of $s$ quarks becomes equilibrated.

\section{B. NS properties}

In order to study NS properties, we have integrated the Tolmann-Oppenheimer-Volkoff (TOV) equations [71,72], together with the differential equations that determine the tidal deformability [73]. The sequence of stars for each hybrid EoS, parametrized by $\left\{\chi_{\omega \omega}, \chi_{\rho}\right\}$ and $\chi_{\omega}=0$, is presented in Fig. 2. Three scenarios are identified: $\chi_{\omega \omega}=$ 10 (left), $\chi_{\omega \omega}=15$ (center), and $\chi_{\omega \omega}=20$ (right). As shown in [50], a larger $\chi_{\omega \omega}$ allows for larger quark branches, capable of reproducing more massive NS with quarks already present inside light NS. If $\chi_{\omega \omega}=10$ and $\chi_{\omega}=0,2 M_{\odot}$ stars are not attained except for large values of $\chi_{\rho}$, where the quark branch is very small. However, for both $\chi_{\omega \omega}=15$ and 20 , it is possible to describe $2 M_{\odot}$ stars with large quark branches. Figure 2 also shows the impact of $\chi_{\rho}$ on the onset of quarks: independently of $\chi_{\omega \omega}$, the larger $\chi_{\rho}$ the larger the quark onset star mass, as expected from the discussion of the pressure behavior. The second effect of a finite $\chi_{\rho}$ is to reduce considerably the quark branch. This is mainly due to the increase in the amount of 


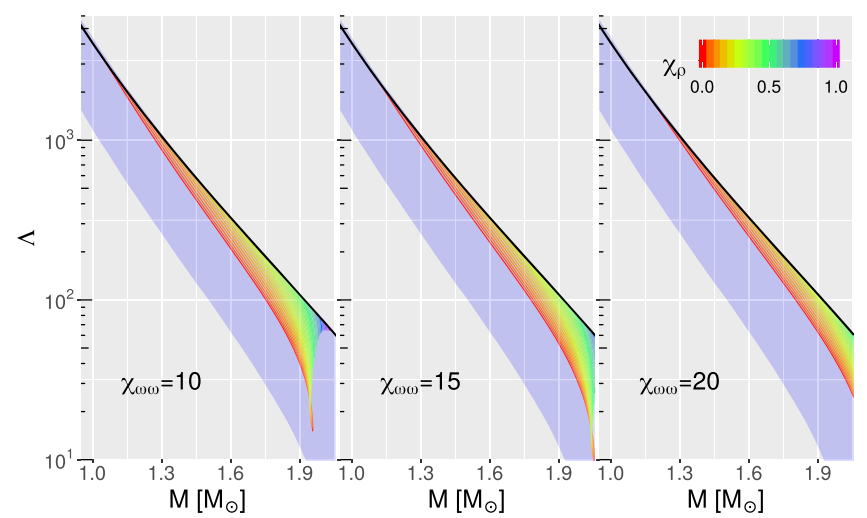

FIG. 3. $\Lambda(M)$ diagrams for $\chi_{\omega \omega}=10$ (left), $\chi_{\omega \omega}=15$ (center), and $\chi_{\omega \omega}=20$ (right). The color scale indicates the $\chi_{\rho}$ value and the solid black line represents the purely hadronic sequence. The background band is the $90 \%$ posterior credible level obtained in [74], when it is imposed that the EoS should describe $1.97 M_{\odot}$ NS.

$s$-quarks that smooths the EoS and therefore, reduces the ability to counterbalance gravity. As a consequence, smaller central densities occur for larger values of $\chi_{\rho}$, as also discussed in [36]. Thus, the radius of the maximum mass, $R_{\max }$, is an increasing function of $\chi_{\rho}$. These results are in accordance with [49], where smaller quark cores were generated by larger $\chi_{\rho}$ values. It is also interesting to realize that the $\chi_{\rho}$ interaction is responsible for the crossing of all NS sequences in a small region of $(M, R)$ diagram. Such crossing is also observed in the pressure and speed of sound of the model for increasing values of $\chi_{\rho}$; see Fig. 1. This effect is related to the decreasing of the onset density of strange quarks with increasing $\chi_{\rho}$ : increasing $\chi_{\rho}$ stiffens the low density region of the EoS while softening its high density region. This opposite behavior in the low and high density regions of the EoS, for increasing $\chi_{\rho}$, generates a crossing point in between these densities regimes. Such behaviors are then translated to the mass-radius relations, giving rise to the crossing behavior observed in Fig. 2 .

The set of hybrid EoS fulfill both the constraints derived from the NICER $x$-ray data for the millisecond pulsar PSR $\mathrm{J} 0030+0451[15,75]$ (shaded rectangular regions) and the confidence intervals of the posterior probability from the LIGO/Virgo analysis [74] for the tidal deformability. Recent results of NICER [76,77], taking into account NICER and other observations, predict for the pulsar MP J0740 +6620 the mass and radius $2.08 \pm 0.07 M_{\odot}$ and $12.35 \pm 0.75 \mathrm{~km} \mathrm{[76],} \mathrm{and} 2.072_{-0.066}^{+0.067} M_{\odot}$ and $12.39_{-0.98}^{+1.30} \mathrm{~km}$ [77], at one standard deviation. Besides, the authors of [76] also predict the radius $12.45 \pm 0.65 \mathrm{~km}$ for a $1.4 M_{\odot}$ star, at one standard deviation. Our results are compatible with these new data.

The emitted gravitational-wave signals from binary NS systems carries information on the tidal deformability of a

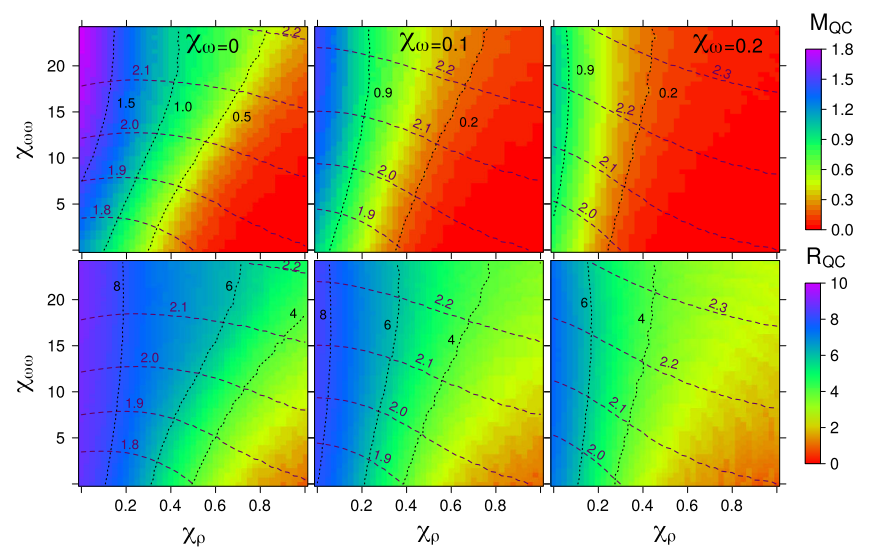

FIG. 4. The quark core mass $M_{\mathrm{QC}}\left[M_{\odot}\right]$ (top) and radius $R_{\mathrm{QC}}$ [km] (bottom) as a function of $\chi_{\omega \omega}$ and $\chi_{\rho}$. The brown dashed lines and black dotted lines represent, respectively, the value of $M_{\max }\left[M_{\odot}\right]$ and specific $\left\{M_{\mathrm{QC}}, R_{\mathrm{QC}}\right\}$ contour lines.

NS, which is an EoS dependent quantity. For a NS with mass $M$, the dimensionless tidal deformability is given by $\Lambda=2 k_{2} R^{5} /\left(3 M^{5}\right)$, where $k_{2}$ is the Love number [73]. The $\Lambda(R)$ diagrams are shown in Fig. 3, for the same couplings defined in Fig. 2. The background band is the $90 \%$ posterior credible level obtained in [74] when it is imposed that the EoS should describe $1.97 M_{\odot}$ stars. Only values $\Lambda(R)$ corresponding to hybrid NS with $M_{\max } \geq 1.97 M_{\odot}$ are shown. The $\Lambda(R)$ determined from the hadron EoS DDME2 lies just at the upper limit of the gray band. All hybrid stars predict values of $\Lambda(R)$ within the band and, as expected, the following trends are observed: the larger $\chi_{\omega \omega}$ and $\chi_{\rho}$ the larger is $\Lambda(R)$, and, therefore, more compressed in the direction of the upper bound of the LIGO/Virgo $90 \%$ posterior credible level.

Let us know investigate how the quark core mass, $M_{\mathrm{QC}}$, and radius, $R_{\mathrm{QC}}$, depend on the couplings $\left\{\chi_{\omega \omega}, \chi_{\rho}, \chi_{\omega}\right\}$. Figure 4 shows the dependence of $M_{\mathrm{QC}}$ (top) and $R_{\mathrm{QC}}$ (bottom) on the interactions $\left\{\chi_{\omega \omega}, \chi_{\rho}\right\}$, for $\chi_{\omega}=0,0.1,0.2$, from the left to the right, respectively. The couplings have a competitive effect: while $\chi_{\omega \omega}$ increases the quark core masses up to $1.53 M_{\odot}$, a finite value $\chi_{\rho}$ or/and $\chi_{\omega}$ has the decreasing effect. The main conclusions drawn are: (i) if $\chi_{\omega}=\chi_{\rho}=0,2 M_{\odot}$ NS are only attained with a value of $\chi_{\omega \omega} \gtrsim 12$ and very large quark cores are possible. As we will see next, these configurations correspond to speed of sound in the center of the star close to 1; (ii) a finite $\chi_{\omega}$ or finite $\chi_{\rho}$ allows $2 M_{\odot}$ NS with intermediate values of $\chi_{\omega \omega}$, however, for the quark cores to have at least one third of the total mass of the star $\chi_{\rho}<0.1$ if $\chi_{\omega}=0.2$, and $\chi_{\rho} \lesssim 0.3$ if $\chi_{\omega}=0.1$. Similar conclusions are drawn concerning quark core radii above $6 \mathrm{~km}$, i.e., above half the star radius. This discussion is in agreement with results in $[49,50]$. To summarize, large quark cores require small values of $\chi_{\rho}$ and $\chi_{\omega}$ and a large $\chi_{\omega \omega}$ coupling. We may ask whether the $\chi_{\rho}$ and the $\chi_{\omega}$ interactions play the same role, or if they 


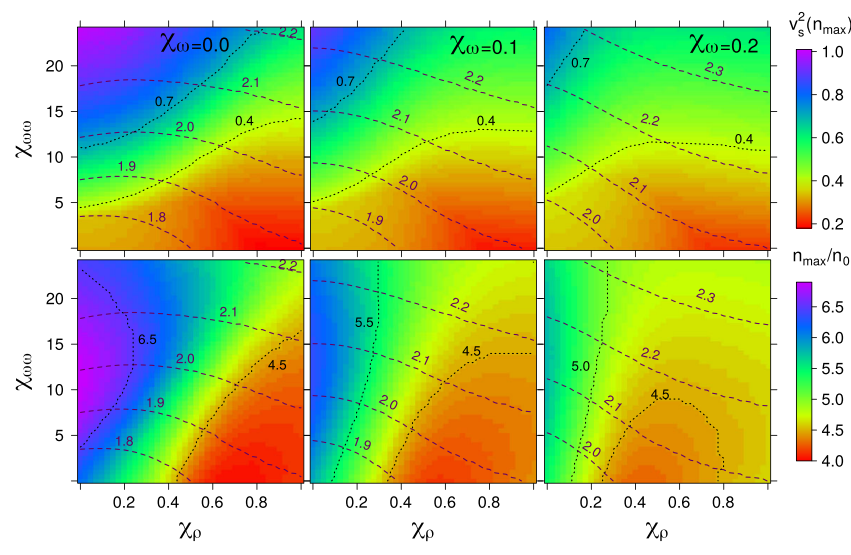

FIG. 5. The squared speed of sound $\left[c^{2}\right]$ (top) and the central densities $\left[n_{0}\right]$ of $M_{\max }$ (bottom) as a function of $\chi_{\omega \omega}$ and $\chi_{\rho}$ for $\chi_{\omega}=0$ (left) 0.1 (center), and 0.2 (right). $n_{0}=0.155 \mathrm{fm}^{-3}$ is the nuclear saturation density. The brown dashed lines and black dotted lines represent, respectively, the value of $M_{\max }\left[M_{\odot}\right]$ and specific $\left\{v_{s}^{2}\left(n_{\max }\right), n_{\max } / n_{0}\right\}$ contour lines.

affect the star properties in a different way. As we will see next the coupling $\chi_{\rho}$ directly affects the amount of strangeness inside the star.

In Fig. 5, we show the $v_{s}^{2}\left(n_{\max }\right)$ (top panels) and $n_{\max }$ (bottom panels), where $n_{\max }$ is the central density of $M_{\max }$, as a function of $\chi_{\omega \omega}$ and $\chi_{\rho}$ for three values of $\chi_{\omega}: 0$ (left), 0.1 (center), and 0.2 (left). There are competitive effects from the three couplings on the $v_{s}^{2}\left(n_{\max }\right)$ : while $v_{s}^{2}$ increases quite rapidly with $\chi_{\omega \omega}$, the $\chi_{\rho}$ channel smooths this trend. This also reflects the fact that a finite value of $\chi_{\rho}$ reduces the central density attained. Notice however, that the largest densities are not attained for the largest values of the coupling $\chi_{\omega \omega}$ : for $\chi_{\rho}=0$ the largest central densities occur with $\chi_{\omega \omega} \sim 12$ and reduce quite fast when $\chi_{\rho}$ increases; for the largest values of $\chi_{\omega \omega}$ considered the reduction of the central density with $\chi_{\rho}$ is slower. As we will see next, this behavior has an effect on the strangeness content of the star. Comparing Figs. 5 and 4, we also conclude that the largest quark cores occur for $\chi_{\omega \omega}>17$ when the central densities are smaller than the maximum value attained for $\chi_{\omega \omega}=12$. The $\chi_{\omega}$ reduces the range of $v_{s}^{2}$ values because it generates smaller quark cores, see Fig. 4 , and the central densities are not large enough for the term $\chi_{\omega \omega}$ to have a strong effect.

Figure 6 represents the fraction of $s$-quarks at the central density of $M_{\max }, Y_{s}^{\max }=\rho_{s}^{\max } / 3 n_{\max }$. The $s$-quark fraction shows a nonlinear dependence on the couplings $\chi_{\omega \omega}$ and $\chi_{\rho}$. We have seen that the larger the value of $\chi_{\rho}$, the earlier the onset of $s$-quarks. On the other hand, a too large $\chi_{\rho}$ does not allow a large quark branch and therefore, large central densities. Large quark branches are possible including a strong $\chi_{\omega \omega}$ coupling. The amount of strangeness in the center of the star results from the interplay of these two effects. As a consequence, the largest central fractions of

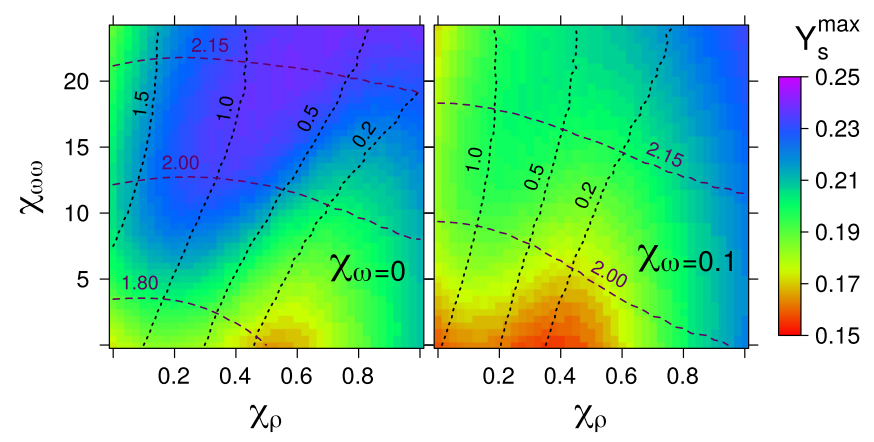

FIG. 6. The fraction of $s$-quarks, $Y_{s}^{\max }$, at the central density of $M_{\max }, n_{\max }$, as a function of $\chi_{\omega \omega}$ and $\chi_{\rho}$ for $\chi_{\omega}=0$ (left) and 0.1 (right). The brown dashed lines and black dotted lines represent, respectively, the value of $M_{\max }\left[M_{\odot}\right]$ and specific $M_{\mathrm{QC}}\left[M_{\odot}\right]$ contour lines.

$s$-quarks happen for large $\chi_{\omega \omega}$ and intermediate $\chi_{\rho}$ values, while the smallest fractions occur for $\chi_{\omega \omega}=0$ : at finite $\chi_{\rho}$ the $s$-quark sets in earlier and with a finite $\chi_{\omega \omega}$ larger central densities are attained and therefore, also larger $s$-quark fractions. The $\chi_{\omega}$ term reduces the maximum central density and therefore, also the central $s$-quark fraction. As a consequence the effect of the $\chi_{\omega \omega}$ term is not so strong. A large amount of $s$-quarks in the star reduces the quark core mass, but it is still possible to have hybrid stars with a quark core with mass of the order of $1 / 3$ to $1 / 2$ of the total star mass. Moreover, these stars with larger amounts of strangeness have a squared speed of sound below 1. Under the conditions studied in the present work the largest fraction of $s$-quark obtained was $25 \%$, for $\chi_{\rho} \approx 0.4$ and $\chi_{\omega \omega}=25$. A larger amount is prevented because a too large $\chi_{\omega \omega}$ gives rise to supraluminous sound speeds. The presence of considerably large fractions of strangeness is essential to allow for phases as the color flavor locked (CFL) superconducting phase.

We include in the Appendix two tables with some properties of the stars obtained with $\chi_{\omega}=0$ and $\chi_{\omega}=$ 0.1 and several values of $\chi_{\omega \omega}$ and $\chi_{\rho}$, respectively, Tables III and IV. These tables include the quark core $\left(M_{\mathrm{QC}}\right)$, quark radii $\left(R_{\mathrm{QC}}\right)$, mass of the heaviest star $\left(M_{\max }\right)$, radii of the heaviest star $\left(R_{\max }\right)$, onset density of quarks $\left(n_{q}\right)$, lightest NS mass with quark content $\left(M_{q}\right)$, central density of the heaviest star $\left(n_{\max }\right)$, strange quark fraction at $n_{\max }\left(Y_{s}^{\max }\right)$, squared speed of sound at $n_{\max }\left[v_{s}^{2}\left(n_{\max }\right)\right]$, radii $\left(R_{1.4 M_{\odot}}\right)$ and tidal deformability $\left(\Lambda_{1.4} M_{\odot}\right)$ of a $1.4 M_{\odot}$ NS. Next, we summarize some of the main conclusions: (i) quark matter sets in low mass stars only if $\chi_{\omega}=0$ and $\chi_{\rho} \lesssim 0.2$. Stars with a mass $\approx 1 M_{\odot}$ may have core quarks; (ii) larger central strangeness fractions are obtained with larger values of $\chi_{\rho}$; (iii) the onset density of quarks increases from $\approx 2 n_{0}$ for $\chi_{\rho}=0$ to $\approx 3 n_{0}$ for $\chi_{\rho}=0.4$; (iv) central densities of maximum mass configuration are not maximum for the largest values of $\chi_{\omega \omega}$, but for a given $\chi_{\rho}$ the maximum mass 


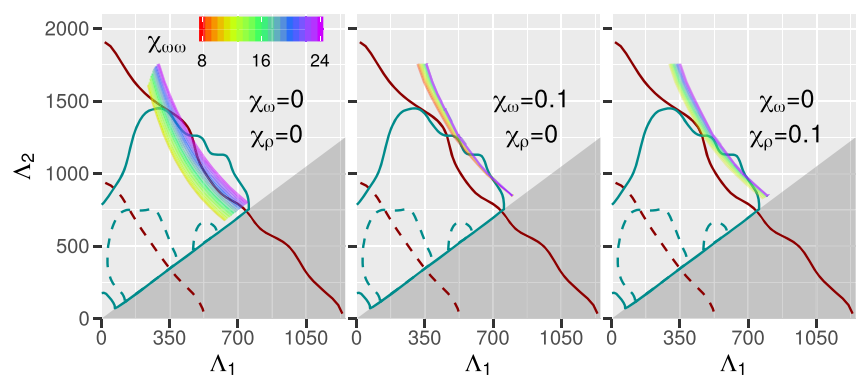

FIG. 7. $\Lambda_{2}-\Lambda_{1}$ diagrams for binary systems with $M_{\text {chirp }}=$ $1.186 M_{\odot}$ and $0.7<q<1$ as a function of $\chi_{\omega \omega}$ (color scale). Three $\left(\chi_{\omega}, \chi_{\rho}\right)$ sets are presented: $(0,0)$ (left), $(0.1,0)$ (center), and $(0,0.1)$ (right). The $50 \%$ (dashed lines) and $90 \%$ (solid lines) credible regions for the marginalized posteriors $\left(\Lambda_{1}, \Lambda_{2}\right)$ from the LIGO/Virgo analysis of the GW170817 event, assuming independent EoS for the NS components (brown) [78] and a parametrized EoS assuming $M_{\max } \geq 1.97 M_{\odot}$ (green) [74].

and maximum $s$-quark central fraction occurs for the largest $\chi_{\omega \omega}$ values; (v) the central speed of sound is maximum for the largest $\chi_{\omega \omega}$ values but decreases when $\chi_{\rho}$ increases; (vi) even with $\chi_{\rho}=0.4$ it is still possible to get a $1 M_{\odot}$ quark core with a central density above $6 n_{0}$; (vii) to attain a maximum mass configuration $\approx 1.97 M_{\odot} \chi_{\omega \omega} \gtrsim 10$.

A finite $\chi_{\rho \rho}$ and/or $\chi_{\omega \rho}$ coupling would not considerably change the above results. A finite $\chi_{\rho \rho}$, within the range considered for $\chi_{\omega \omega}$, i.e., between 0 and 25 , would increase the $Y_{s}^{\max }$ up to $1 \%$, while both $M_{\max }$ and $v_{s}^{2}\left(n_{\max }\right)$ are almost insensitive. A finite value $\chi_{\rho \rho}$ (in the same range) would originate an increase of $1 \%$ in $M_{\max }$, an increase up to $9 \%$ in $Y_{s}^{\max }$, and a reduction up to $20 \%$ in $v_{s}^{2}\left(n_{\max }\right)$. Therefore, by including the $\chi_{\omega \rho}$ term we could have at most $\approx 28 \% s$-quarks for $\chi_{\omega \omega}=25$ and $\chi_{\rho}=0.5$. However, the reduction of the speed of sound, originated from $\chi_{\rho \rho}$, is interesting as it allows us to counterbalance the $\chi_{\omega \omega}$ impact on the speed of sound. A CFL phase is favored when the three flavors appear in similar amounts. It could be that more favorable conditions for a CFL phase are still possible with a correct choice of the couplings $\chi_{\omega \omega}$, that increases the quark branch and speed of sound, and $\chi_{\omega \rho}$ that increases the strangeness content and decreases the speed of sound. This interplay effect will be explored in a future work.

\section{Binary tidal deformability}

The leading tidal parameter of the gravitational-wave signal from a NS merger is the effective tidal deformability,

$$
\tilde{\Lambda}=\frac{16}{13} \frac{(12 q+1) \Lambda_{1}+(12+q) q^{4} \Lambda_{2}}{(1+q)^{5}},
$$

where $q=M_{2} / M_{1}<1$ is the binary mass ratio [73]. The $\Lambda_{1}\left(M_{1}\right)$ and $\Lambda_{2}\left(M_{2}\right)$ represent the tidal deformability (mass) of the primary and the secondary NS in the binary, respectively. The GW170817 event provides $\tilde{\Lambda}=300_{-230}^{+420}$
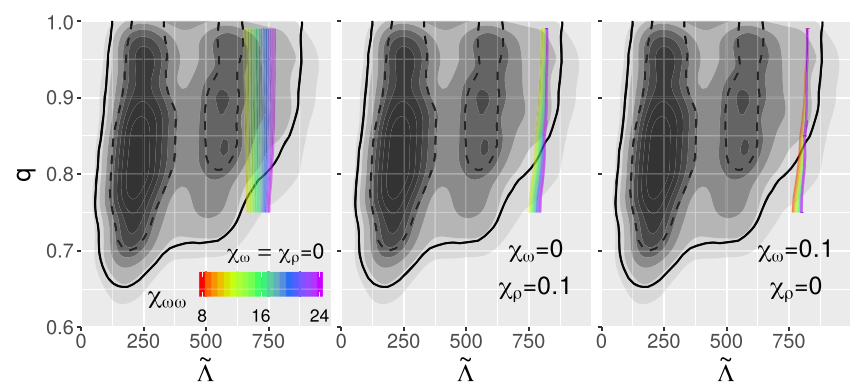

FIG. 8. $q-\tilde{\Lambda}$ diagrams for binary systems with $M_{\text {chirp }}=$ $1.186 M_{\odot}$ as a function of $\chi_{\omega \omega}$ (color scale). Three $\left(\chi_{\omega}, \chi_{\rho}\right)$ sets are presented: $(0,0)$ (left), $(0.1,0)$ (center), and $(0,0.1)$ (right). The LIGO/Virgo Collaboration results [78] for the probability distribution function of the joint posterior is shown by the gray gradient, and the black dashed and solid lines indicate the $50 \%$ and $90 \%$ credible regions, respectively.

(90\% credible interval) and $0.73 \leq q \leq 1$ for the binary mass ratio [78]. The chirp mass of the binary system, $M_{\text {chirp }}=\left(M_{1} M_{2}\right)^{3 / 5} /\left(M_{1}+M_{2}\right)^{1 / 5}$, is measured with a good accuracy during the gravitational wave detection. For the GW170817 event, it was measured to be $1.186_{-0.001}^{+0.001} M_{\odot}$ [78].

In the following, we fix the chirp mass as $M_{\text {chirp }}=$ $1.186 M_{\odot}$ and determine $\Lambda_{1,2}$ for binary systems of mass ratios $0.73 \leq q \leq 1$. The $\Lambda_{2}-\Lambda_{1}$ diagrams are shown in Fig. 7 as a function of $\chi_{\omega \omega}$ (color scale) for three $\left(\chi_{\omega}, \chi_{\rho}\right)$ sets: $(0,0)$ (left), $(0.1,0)$ (center), and $(0,0.1)$ (right). We also show the two distinct credible regions from LIGO/Virgo analysis: assuming that each NS has a different EoS, and $\Lambda_{1,2}$ vary independently (brown lines), and using a parametrized EoS with the assumption of $M_{\max } \geq 1.97 M_{\odot}$ (dark green lines). All hybrid EoS for the $\left(\chi_{\omega}=0, \chi_{\rho}=0\right)$ case fall inside the $90 \%$ credible region for mass ratios $0.8 \leq q \leq 1$. For highly asymmetric binaries, $0.73 \leq q<$ 0.8 , the primary mass $M_{1}$ can be as large as $1.60 M_{\odot}$ while $M_{2}$ (secondary mass) can be as low as $1.17 M_{\odot}$. The considerable large radius of $M_{1}$ for parametrizations with large $\chi_{\rho}$, very close to the purely hadronic EoS result (see solid black line in Fig. 2), results in large $\tilde{\Lambda}$ compared with the LIGO/Virgo analysis (note that we are only considering EoS that reach $\left.1.97 M_{\odot}\right)$. We see that for $\left(\chi_{\omega}=0, \chi_{\rho}=0\right)$ all EoS are compatible with LIGO/Virgo 90\% credible region. A small and finite $\chi_{\omega}$ and/or $\chi_{\rho}$ reduces the quark core and thus gives larger values in the $\Lambda_{1}-\Lambda_{2}$ diagram. The configurations $\left(\chi_{\omega}, \chi_{\rho}\right)=(0.1,0)$ (center) and $(0,0.1)$ (right) still predict a large number of hybrid NS compatible with the $90 \%$ credible region of LIGO/Virgo, for $q \gtrsim 0.85$.

We compare our set of hybrid EoS and the probability density function (pdf) $P(q, \tilde{\Lambda})$ from the LIGO/ Virgo analysis in Fig. 8 (the same sets as in Fig. 7). Marginalizing the pdf over $q$, i.e., $P(\tilde{\Lambda})=\int P(q, \tilde{\Lambda}) d q$, 
results in a pdf for $\tilde{\Lambda}$ characterized by $\tilde{\Lambda}=300_{-190}^{+500}$ [78]. We see that $\tilde{\Lambda}$ depends very weakly on the binary mass ratio $q$. Another important observation is that all hybrid EoS shown are within the $90 \%$ credible interval for $q \gtrsim 0.8$ for $\chi_{\omega}=\chi_{\rho}=0$ and $q \gtrsim 0.85$ if $\left(\chi_{\omega}, \chi_{\rho}\right)=(0.1,0)$ or $(0,0.1)$. The EoS are concentrated around $\tilde{\Lambda}=650-750$ for $\left(\chi_{\omega}=0, \chi_{\rho}=0\right)$, and $\tilde{\Lambda} \sim 800$ for the other two cases. These results show that all hybrid stars studied, both with small or large quark cores which is controlled by the $\chi_{\omega \omega}$ coupling, are compatible with the GW170817 event.

\section{CONCLUSIONS}

We have analyzed the effect of several channel interactions in hadron-quark hybrid EoS within the three-flavor NJL model. Each hybrid EoS consists of charge-neutral matter in $\beta$-equilibrium that has a phase transition from hadronic to quark matter. We have studied how the stability of hybrid star sequences and their properties depend on the coupling values. The interaction coupling considered in this work were: $\chi_{\omega}, \chi_{\omega \omega}, \chi_{\rho}, \chi_{\rho \rho}$, and $\chi_{\omega \rho}$. Our analysis showed, however, a weak impact of both $\chi_{\rho \rho}$ and $\chi_{\omega \rho}$ interactions on the quark EoS, in particular of the first one. Therefore, we focused our discussion on the $\chi_{\omega}, \chi_{\omega \omega}$ and $\chi_{\rho}$ channels, and presented a brief estimate of the effect of the couplings $\chi_{\rho \rho}$ and $\chi_{\omega \rho}$. As already seen in [50], $\chi_{\omega \omega}$ considerably changes the $v_{s}(n)$ dependence, which turns out to be crucial in supporting massive hybrid NS. On the other hand, $\chi_{\rho}$ controls the onset density of the strange quark, larger values giving lower onset densities and, consequently, at moderate baryonic densities give rise to a decrease of the speed of sound.

These effects on the quark EoS are reflected on the properties of hybrid stars in the following manner: (a) the $\chi_{\omega \omega}$ is responsible for longer quark branches in the $M(R)$ diagram, larger central baryonic densities, the onset of quarks in light mass NS and the possibility of sustaining these quark cores in massive stars with masses up to $\sim 2.1 M_{\odot}$; (b) the $\chi_{\rho}$ coupling mainly controls the onset of strangeness in the star, a earlier onset occurring with a larger coupling, and its strangeness content, larger $s$-quark fractions occur for larger couplings. The central speed of sound decreases as $\chi_{\rho}$ increases. $\chi_{\rho}$ also influences the onset of quarks, a larger coupling pushes the onset to larger densities, decreases the size of the quark branch and gives rise to larger radii. Besides, this interaction is responsible for a small crossing region in the $(M, R)$ diagram taking all the EoS with different $\chi_{\rho}$ couplings and the other couplings fixed; (c) the $\chi_{\omega}$ is mainly responsible to increasing the maximum mass in the $(M, R)$ diagram while pushing the quark onset to larger densities. This gives rise to shorter quark branches, smaller central baryonic densities and smaller strangeness fractions.
Both $\chi_{\omega \omega}$ and $\chi_{\rho}$ have a competitive effect on the size of quark cores. While $\chi_{\omega \omega}$ is able to generate quark core masses up to $1.53 M_{\odot}$, a finite $\chi_{\rho}$ decreases this effect. However, moderate values $\chi_{\rho}$ are seen to describe heavier hybrid NS, with smaller quark cores but with larger strangeness fractions and lower speed of sound at the NS central densities. In particular, it is possible to have quark cores with masses of the order of $0.8-1 M_{\odot}$ and radii of the order of $6 \mathrm{~km}$, i.e., one third of the star radius. In other words, $\chi_{\rho}$ is capable of producing massive hybrid NS, and although with smaller quark cores than taking $\chi_{\rho}=0$ and a larger $\chi_{\omega \omega}$, with considerable lower values for $v_{s}^{2}$ at the star center. For any coupling $\chi_{\rho}>0.25$, regardless of the $\chi_{\omega \omega}$ value, any quark core in the hybrid stars sequences contains strange quarks since the onset density of the strange quarks happens at lower values than the transition from hadronic to quark matter. Under the conditions investigated in the present work, a maximum $s$-quark fraction of $25 \%$ was obtained for a star with a quark core mass having a third of the total star mass. Including a $\chi_{\omega \rho}$ term this fraction could rise up to $28 \%$.

We have shown that the presently existing mass and tidal deformability constraints from NS observations allow for the existence of hybrid stars with a large strangeness content and large quark cores. In order to get large $s$-quark content, the isovector-vector interaction was included. The hybrid stars were built starting from an hadronic EoS that satisfy presently accepted nuclear matter properties, DDME2 and considering a quark model that is constrained to the vacuum properties of several mesons. Under the conditions considered no twin stars [34] have been found. In [34], twin stars where obtained within a two-flavor NJL model with four-quark and eight-quark terms, and, besides the scalar channels also isoscalar-vector channels were included.

We have compared our set of hybrid stars with the analysis from the GW170817 event. A considerable subset of EoS satisfy the $90 \%$ credible region for $\tilde{\Lambda}$. The $\chi_{\omega \omega}$ is able to generate massive quark cores, already present in $1.4 M_{\odot}$ stars, with $\tilde{\Lambda}<800$, even for finite but small values of both $\chi_{\rho}$ and $\chi_{\omega}$. The recently reported GW190814 event of a compact binary coalescence showed that while the primary component is conclusively a black hole, the secondary component with mass of 2.50-2.67 $M_{\odot}$ remains yet inconclusive [14]. In the present work, we cannot describe such a massive NS as an hybrid star described by the present quark model. However, our results are dependent on the hadronic EoS considered and changing the hadronic part of the hybrid EoS might make the EoS hard enough to reach such high mass hybrid stars [49]. In this case, the quark content of such stars will be very small and the tidal deformability of stars with low masses, within such models will be given by the hadronic sector of the EoS. 


\section{ACKNOWLEDGMENTS}

This work was partially supported by national funds from FCT (Fundação para a Ciência e a Tecnologia, I.P, Portugal) under the IDPASC Ph.D. program (International Doctorate Network in Particle Physics, Astrophysics and Cosmology), with the Grant No. PD/BD/128234/2016 (R. C. P.), under the Projects No. UID/FIS/04564/2019, No. UID/04564/2020, and No. POCI-01-0145-FEDER-
029912 with financial support from Science, Technology and Innovation, in its FEDER component, and by the FCT/ MCTES budget through national funds (OE).

\section{APPENDIX: HYBRID STARS PROPERTIES}

We summarize some properties of the hybrid NS obtained with $\chi_{\omega}=0$ and $\chi_{\omega}=0.1$ in Tables III and IV, respectively, for several values of $\chi_{\omega \omega}$ and $\chi_{\rho}$.

TABLE III. Several NS properties for each different hybrid $\operatorname{EoS}\left(\chi_{\rho}, \chi_{\omega \omega}, \chi_{\omega}=0\right)$ : quark core $\left(M_{\mathrm{QC}}\right)$, quark radii $\left(R_{\mathrm{QC}}\right)$, mass of the heaviest star $\left(M_{\max }\right)$, radii of the heaviest star $\left(R_{\max }\right)$, onset density of quarks $\left(n_{q}\right)$, lightest NS mass with quark content $\left(M_{q}\right)$, central density of the heaviest $\operatorname{star}\left(n_{\max }\right)$, strange quark fraction at $n_{\max }\left(Y_{s}^{\max }\right)$, squared speed of sound at $n_{\max }\left[v_{s}^{2}\left(n_{\max }\right)\right]$, radii $\left(R_{1.4} M_{\odot}\right)$ and tidal deformability $\left(\Lambda_{1.4 M_{\odot}}\right)$ of a $1.4 M_{\odot}$ NS.

\begin{tabular}{|c|c|c|c|c|c|c|c|c|c|c|c|c|}
\hline & & $M_{\mathrm{QC}}$ & $R_{\mathrm{QC}}$ & $M_{\max }$ & $R_{\max }$ & $n_{q}$ & $M_{q}$ & $n_{\max }$ & $Y_{s}^{\max }$ & $v_{s}^{2}\left(n_{\max }\right)$ & $R_{1.4 M_{\odot}}$ & $\Lambda_{1.4 M_{\odot}}$ \\
\hline$\chi_{\rho}$ & $\chi_{\omega \omega}$ & {$\left[M_{\odot}\right]$} & {$[\mathrm{km}]$} & {$\left[M_{\odot}\right]$} & {$[\mathrm{km}]$} & {$\left[\mathrm{fm}^{-3}\right]$} & {$\left[M_{\odot}\right]$} & {$\left[\mathrm{fm}^{-3}\right]$} & & {$\left[\mathrm{c}^{2}\right]$} & {$[\mathrm{km}]$} & \\
\hline 0.0 & 0 & 1.21 & 8.69 & 1.71 & 11.69 & 0.33 & 0.92 & 0.95 & 0.17 & 0.32 & 12.54 & 452 \\
\hline 0.0 & 5 & 1.41 & 8.77 & 1.84 & 11.31 & 0.33 & 0.98 & 1.03 & 0.20 & 0.43 & 12.68 & 493 \\
\hline 0.0 & 10 & 1.57 & 8.82 & 1.96 & 11.03 & 0.34 & 1.04 & 1.07 & 0.20 & 0.66 & 12.81 & 538 \\
\hline 0.0 & 15 & 1.68 & 8.89 & 2.05 & 10.96 & 0.34 & 1.10 & 1.06 & 0.20 & 0.83 & 12.91 & 578 \\
\hline 0.0 & 20 & 1.74 & 8.96 & 2.13 & 11.01 & 0.35 & 1.18 & 1.03 & 0.2 & 0.93 & 13.02 & 621 \\
\hline 0.2 & 0 & 0.76 & 7.24 & 1.72 & 12.26 & 0.39 & 1.38 & 0.84 & 0.18 & 0.31 & 13.19 & 690 \\
\hline 0.2 & 5 & 0.97 & 7.55 & 1.84 & 11.87 & 0.40 & 1.46 & 0.93 & 0.21 & 0.37 & 13.20 & 705 \\
\hline 0.2 & 10 & 1.20 & 7.78 & 1.95 & 11.43 & 0.41 & 1.53 & 1.00 & 0.22 & 0.61 & 13.20 & 705 \\
\hline 0.2 & 15 & 1.32 & 7.89 & 2.04 & 11.25 & 0.42 & 1.63 & 1.02 & 0.23 & 0.78 & 13.20 & 705 \\
\hline 0.2 & 20 & 1.37 & 7.92 & 2.12 & 11.26 & 0.43 & 1.71 & 1.00 & 0.22 & 0.89 & 13.20 & 705 \\
\hline 0.4 & 0 & 0.30 & 5.09 & 1.77 & 12.82 & 0.48 & 1.71 & 0.72 & 0.17 & 0.29 & 13.20 & 705 \\
\hline 0.4 & 5 & 0.47 & 5.78 & 1.86 & 12.52 & 0.49 & 1.77 & 0.79 & 0.20 & 0.31 & 13.20 & 705 \\
\hline 0.4 & 10 & 0.75 & 6.52 & 1.95 & 12.02 & 0.50 & 1.84 & 0.89 & 0.22 & 0.50 & 13.20 & 705 \\
\hline 0.4 & 15 & 0.95 & 6.88 & 2.05 & 11.68 & 0.51 & 1.91 & 0.94 & 0.23 & 0.69 & 13.20 & 705 \\
\hline 0.4 & 20 & 1.03 & 7.01 & 2.13 & 11.59 & 0.52 & 1.98 & 0.94 & 0.23 & 0.82 & 13.20 & 705 \\
\hline
\end{tabular}

TABLE IV. Several NS properties for each different hybrid EoS $\left(\chi_{\rho}, \chi_{\omega \omega}, \chi_{\omega}=0.1\right)$ : quark core $\left(M_{\mathrm{QC}}\right)$, quark radii $\left(R_{\mathrm{QC}}\right)$, mass of the heaviest star $\left(M_{\max }\right)$, radii of the heaviest star $\left(R_{\max }\right)$, onset density of quarks $\left(n_{q}\right)$, lightest NS mass with quark content $\left(M_{q}\right)$, central density of the heaviest $\operatorname{star}\left(n_{\max }\right)$, strange quark fraction at $n_{\max }\left(Y_{s}^{\max }\right)$, squared speed of sound at $n_{\max }\left[v_{s}^{2}\left(n_{\max }\right)\right]$, radii $\left(R_{1.4} M_{\odot}\right)$ and tidal deformability $\left(\Lambda_{1.4 M_{\odot}}\right)$ of a $1.4 M_{\odot}$ NS.

\begin{tabular}{lrcccccccccc}
\hline \hline & & $M_{\mathrm{QC}}$ & $R_{\mathrm{QC}}$ & $M_{\max }$ & $R_{\max }$ & $n_{q}$ & $M_{q}$ & $n_{\max }$ & $Y_{s}^{\max }$ & $v_{s}^{2}\left(n_{\max }\right)$ & $R_{1.4 M_{\odot}} \Lambda_{1.4 M_{\odot}}$ \\
\hline$\chi_{\rho}$ & $\chi_{\omega \omega}$ & {$\left[M_{\odot}\right]$} & {$[\mathrm{km}]$} & {$\left[M_{\odot}\right]$} & {$[\mathrm{km}]$} & {$\left[\mathrm{fm}^{-3}\right]$} & {$\left[M_{\odot}\right]$} & {$\left[\mathrm{fm}^{-3}\right]$} & & {$\left[\mathrm{c}^{2}\right]$} & {$[\mathrm{km}]$} \\
\hline 0.0 & 0 & 1.03 & 7.99 & 1.80 & 11.99 & 0.37 & 1.27 & 0.90 & 0.16 & 0.33 & 13.07 \\
0.0 & 5 & 1.21 & 8.20 & 1.91 & 11.71 & 0.38 & 1.34 & 0.95 & 0.17 & 0.40 & 13.16 \\
0.0 & 10 & 1.35 & 8.29 & 2.01 & 11.48 & 0.39 & 1.42 & 0.98 & 0.18 & 0.58 & 13.20 \\
0.0 & 15 & 1.44 & 8.35 & 2.10 & 11.39 & 0.40 & 1.50 & 0.98 & 0.18 & 0.73 & 13.20 \\
0.0 & 20 & 1.49 & 8.37 & 2.17 & 11.39 & 0.41 & 1.59 & 0.97 & 0.18 & 0.84 & 13.20 \\
0.2 & 0 & 0.51 & 6.15 & 1.84 & 12.59 & 0.45 & 1.69 & 0.78 & 0.16 & 0.32 & 13.20 \\
0.2 & 5 & 0.66 & 6.53 & 1.94 & 12.35 & 0.46 & 1.76 & 0.83 & 0.18 & 0.34 & 13.20 \\
0.2 & 10 & 0.82 & 6.82 & 2.02 & 12.08 & 0.47 & 1.84 & 0.87 & 0.19 & 0.49 & 13.20 \\
0.2 & 15 & 0.93 & 6.98 & 2.11 & 11.9 & 0.49 & 1.92 & 0.9 & 0.2 & 0.64 & 13.20 \\
0.2 & 20 & 0.98 & 7.00 & 2.18 & 11.83 & 0.51 & 2.0 & 0.9 & 0.2 & 0.75 & 13.20 \\
0.4 & 0 & 0.14 & 3.84 & 1.91 & 13.07 & 0.54 & 1.88 & 0.67 & 0.16 & 0.3 & 13.20 \\
0.4 & 5 & 0.22 & 4.47 & 1.98 & 12.92 & 0.55 & 1.95 & 0.71 & 0.17 & 0.31 & 705 \\
0.4 & 10 & 0.35 & 5.11 & 2.06 & 12.7 & 0.56 & 2.01 & 0.75 & 0.19 & 0.39 & 13.20 \\
0.4 & 15 & 0.48 & 5.57 & 2.13 & 12.48 & 0.57 & 2.08 & 0.79 & 0.2 & 0.52 & 705 \\
0.4 & 20 & 0.57 & 5.81 & 2.2 & 12.32 & 0.58 & 2.14 & 0.81 & 0.2 & 0.64 & 13.20 \\
\hline \hline
\end{tabular}


[1] P. Demorest, T. Pennucci, S. Ransom, M. Roberts, and J. Hessels, Nature (London) 467, 1081 (2010).

[2] Z. Arzoumanian et al. (NANOGrav Collaboration), Astrophys. J. Suppl. 235, 37 (2018).

[3] J. Antoniadis et al., Science 340, 1233232 (2013).

[4] H. T. Cromartie, E. Fonseca, S. M. Ransom, P. B. Demorest, Z. Arzoumanian, H. Blumer, P. R. Brook, M. E. DeCesar, T. Dolch, and J. A. Ellis et al., Nat. Astron., 4, 72 (2020).

[5] B. P. Abbott et al. (LIGO Scientific and Virgo Collaborations), Phys. Rev. Lett. 119, 161101 (2017).

[6] B. A. et al. (The LIGO Scientific and Virgo Collaborations), Phys. Rev. Lett. 121, 161101 (2018).

[7] B. P. Abbott et al. (LIGO Scientific, Virgo, Fermi-GBM, and INTEGRAL Collaborations), Astrophys. J. 848, L13 (2017).

[8] B. P. Abbott et al. (LIGO Scientific, Virgo, Fermi GBM, INTEGRAL, IceCube, AstroSat Cadmium Zinc Telluride Imager Team, IPN, Insight-Hxmt, ANTARES, Swift, AGILE Team, 1M2H Team, Dark Energy Camera GWEM, DES, DLT40, GRAWITA, Fermi-LAT, ATCA, ASKAP, Las Cumbres Observatory Group, OzGrav, DWF (Deeper Wider Faster Program), AST3, CAASTRO, VINROUGE, MASTER, J-GEM, GROWTH, JAGWAR, CaltechNRAO, TTU-NRAO, NuSTAR, Pan-STARRS, MAXI Team, TZAC Consortium, KU, Nordic Optical Telescope, ePESSTO, GROND, Texas Tech University, SALT Group, TOROS, BOOTES, MWA, CALET, IKIGW Follow-up, H.E.S.S., LOFAR, LWA, HAWC, Pierre Auger, ALMA, Euro VLBI Team, Pi of Sky, Chandra Team at McGill University, DFN, ATLAS Telescopes, High Time Resolution Universe Survey, RIMAS, RATIR, SKA South Africa/MeerKAT Collaborations), Astrophys. J. 848, L12 (2017).

[9] D. Radice, S. Bernuzzi, W. Del Pozzo, L. F. Roberts, and C. D. Ott, Astrophys. J. 842, L10 (2017).

[10] D. Radice, A. Perego, F. Zappa, and S. Bernuzzi, Astrophys. J. 852, L29 (2018).

[11] A. Bauswein, N.-U. Friedrich Bastian, D. Blaschke, K. Chatziioannou, J. A. Clark, T. Fischer, H.-T. Janka, O. Just, M. Oertel, and N. Stergioulas, AIP Conf. Proc. 2127, 020013 (2019).

[12] M. W. Coughlin et al., Mon. Not. R. Astron. Soc. 480, 3871 (2018).

[13] Y.-Z. Wang, D.-S. Shao, J.-L. Jiang, S.-P. Tang, X.-X. Ren, F.-W. Zhang, Z.-P. Jin, Y.-Z. Fan, and D.-M. Wei, Astrophys. J. 877, 2 (2019).

[14] R. Abbott et al. (LIGO Scientific and Virgo Collaborations), Astrophys. J. Lett. 896, L44 (2020).

[15] T. E. Riley et al., Astrophys. J. Lett. 887, L21 (2019).

[16] B. P. Abbott et al. (Virgo and LIGO Scientific Collaborations), Phys. Rev. Lett. 121, 161101 (2018).

[17] M. G. Alford, S. Han, and K. Schwenzer, J. Phys. G 46, 114001 (2019).

[18] E. R. Most, L. J. Papenfort, V. Dexheimer, M. Hanauske, S. Schramm, H. Stöcker, and L. Rezzolla, Phys. Rev. Lett. 122, 061101 (2019).

[19] L. R. Weih, M. Hanauske, and L. Rezzolla, Phys. Rev. Lett. 124, 171103 (2020).

[20] T. Hatsuda and T. Kunihiro, Phys. Rep. 247, 221 (1994).

[21] M. Buballa, Phys. Rep. 407, 205 (2005).
[22] K. Schertler, S. Leupold, and J. Schaffner-Bielich, Phys. Rev. C 60, 025801 (1999).

[23] M. Hanauske, L. M. Satarov, I. N. Mishustin, H. Stoecker, and W. Greiner, Phys. Rev. D 64, 043005 (2001).

[24] M. Baldo, M. Buballa, F. Burgio, F. Neumann, M. Oertel, and H. Schulze, Phys. Lett. B 562, 153 (2003).

[25] D. Menezes and C. Providencia, Phys. Rev. C 68, 035804 (2003).

[26] G. Pagliara and J. Schaffner-Bielich, Phys. Rev. D 77, 063004 (2008).

[27] L. Bonanno and A. Sedrakian, Astron. Astrophys. 539, A16 (2012).

[28] C. H. Lenzi and G. Lugones, Astrophys. J. 759, 57 (2012).

[29] K. Masuda, T. Hatsuda, and T. Takatsuka, Prog. Theor. Exp. Phys. 2013, 073D01 (2013).

[30] T. Klähn, R. Łastowiecki, and D. B. Blaschke, Phys. Rev. D 88, 085001 (2013).

[31] D. Logoteta, C. Providência, and I. Vidaña, Phys. Rev. C 88, 055802 (2013).

[32] N. Glendenning, Compact Stars: Nuclear Physics, Particle Physics and General Relativity, Astronomy and Astrophysics Library (Springer, New York, 2012).

[33] S. Benic, Eur. Phys. J. A 50, 111 (2014).

[34] S. Benic, D. Blaschke, D. E. Alvarez-Castillo, T. Fischer, and S. Typel, Astron. Astrophys. 577, A40 (2015).

[35] A. Zacchi, M. Hanauske, and J. Schaffner-Bielich, Phys. Rev. D 93, 065011 (2016).

[36] R. Câmara Pereira, P. Costa, and C. Providência, Phys. Rev. D 94, 094001 (2016).

[37] X. Wu, A. Ohnishi, and H. Shen, Phys. Rev. C 98, 065801 (2018).

[38] N. Yasutake, R. Lastowiecki, S. Benic, D. Blaschke, T. Maruyama, and T. Tatsumi, Phys. Rev. C 89, 065803 (2014).

[39] G. Pagliara and J. Schaffner-Bielich, Phys. Rev. D 77, 063004 (2008).

[40] A. A. Osipov, B. Hiller, and J. da Providencia, Phys. Lett. B 634, 48 (2006).

[41] A. A. Osipov, B. Hiller, A. H. Blin, and J. da Providencia, Ann. Phys. (Amsterdam) 322, 2021 (2007).

[42] D. Alvarez-Castillo, A. Ayriyan, S. Benic, D. Blaschke, H. Grigorian, and S. Typel, Eur. Phys. J. A 52, 69 (2016).

[43] D. Alvarez-Castillo, S. Benic, D. Blaschke, S. Han, and S. Typel, Eur. Phys. J. A 52, 232 (2016).

[44] I. F. Ranea-Sandoval, S. Han, M. G. Orsaria, G. A. Contrera, F. Weber, and M. G. Alford, Phys. Rev. C 93, 045812 (2016).

[45] M. G. Alford, S. Han, and M. Prakash, Phys. Rev. D 88, 083013 (2013).

[46] M. G. Alford and S. Han, Eur. Phys. J. A 52, 62 (2016).

[47] S. Han, M. A. A. Mamun, S. Lalit, C. Constantinou, and M. Prakash, Phys. Rev. D 100, 103022 (2019).

[48] E. Annala, T. Gorda, A. Kurkela, J. Nättilä, and A. Vuorinen, Nat. Phys. 16, 907 (2020).

[49] M. Ferreira, R. Câmara Pereira, and C. Providência, Phys. Rev. D 101, 123030 (2020).

[50] M. Ferreira, R. Câmara Pereira, and C. Providência, Phys. Rev. D 102, 083030 (2020).

[51] S. P. Klevansky, Rev. Mod. Phys. 64, 649 (1992). 
[52] I. Vidana, D. Logoteta, C. Providencia, A. Polls, and I. Bombaci, Europhys. Lett. 94, 11002 (2011).

[53] I. Bednarek, P. Haensel, J. L. Zdunik, M. Bejger, and R. Manka, Astron. Astrophys. 543, A157 (2012).

[54] S. Weissenborn, D. Chatterjee, and J. Schaffner-Bielich, Nucl. Phys. A881, 62 (2012).

[55] S. Weissenborn, D. Chatterjee, and J. Schaffner-Bielich, Phys. Rev. C 85, 065802 (2012); 90, 019904(E) (2014).

[56] C. Providencia and A. Rabhi, Phys. Rev. C 87, 055801 (2013).

[57] M. Oertel, C. Providência, F. Gulminelli, and A. R. Raduta, J. Phys. G 42, 075202 (2015).

[58] M. Fortin, C. Providencia, A. Raduta, F. Gulminelli, J. L. Zdunik, P. Haensel, and M. Bejger, Phys. Rev. C 94, 035804 (2016).

[59] D. Chatterjee and I. Vidaña, Eur. Phys. J. A 52, 29 (2016).

[60] V. Dexheimer, R. O. Gomes, T. Klähn, S. Han, and M. Salinas, Phys. Rev. C 103, 025808 (2021).

[61] A. Li, Z. Miao, S. Han, and B. Zhang, arXiv:2103.15119.

[62] J. Morais, B. Hiller, and A. A. Osipov, Phys. Rev. D 95, 074033 (2017).

[63] R. Câmara Pereira, J. Moreira, and P. Costa, Eur. Phys. J. A 56, 214 (2020).

[64] K. A. Olive et al. (Particle Data Group), Chin. Phys. C 38, 090001 (2014).
[65] T. Klahn, D. Blaschke, F. Sandin, C. Fuchs, A. Faessler, H. Grigorian, G. Ropke, and J. Trumper, Phys. Lett. B 654, 170 (2007).

[66] D. P. Menezes, M. B. Pinto, L. B. Castro, P. Costa, and C. Providência, Phys. Rev. C 89, 055207 (2014).

[67] T. Klahn and T. Fischer, Astrophys. J. 810, 134 (2015).

[68] G. A. Lalazissis, T. Nikšić, D. Vretenar, and P. Ring, Phys. Rev. C 71, 024312 (2005).

[69] M. Dutra, O. Lourenço, S. Avancini, B. Carlson, A. Delfino, D. Menezes, C. Providência, S. Typel, and J. Stone, Phys. Rev. C 90, 055203 (2014).

[70] K. Hebeler, J. Lattimer, C. Pethick, and A. Schwenk, Astrophys. J. 773, 11 (2013).

[71] R. C. Tolman, Phys. Rev. 55, 364 (1939).

[72] J. R. Oppenheimer and G. M. Volkoff, Phys. Rev. 55, 374 (1939).

[73] T. Hinderer, B. D. Lackey, R. N. Lang, and J. S. Read, Phys. Rev. D 81, 123016 (2010).

[74] B. P. Abbott et al. (LIGO Scientific and Virgo Collaborations), Phys. Rev. Lett. 121, 161101 (2018).

[75] M. Miller et al., Astrophys. J. Lett. 887, L24 (2019).

[76] T. E. Riley et al., arXiv:2105.06980.

[77] M. C. Miller et al., arXiv:2105.06979.

[78] B. P. Abbott et al. (LIGO Scientific and Virgo Collaborations), Phys. Rev. X 9, 011001 (2019). 